\title{
Xanthoma Disseminatum Presenting as Liver Failure in an Adult: A Case Report
}

\author{
Maxwell Bressman*, Thomas Stringer, Emily Hoffman and Matthew Shaines \\ Department of Medicine, Montefiore Medical Center, Division of Cardiology, Bronx, NY, USA \\ *Corresponding author: Maxwell Bressman, Department of Medicine, Montefiore Medical Center, Division of Cardiology, \\ Bronx, NY, USA
}

\section{ARTICLE INFO}

Received: 幽 February 25, 2021

Published: March 04, 2021

Citation: Maxwell B, Thomas S, Emily $\mathrm{H}$ Matthew S. Xanthoma Disseminatum Presenting as Liver Failure in an Adult: A Case Report. Biomed J Sci \& Tech Res 34(2)2021. BJSTR. MS.ID.005539.

Keywords: Xanthoma Disseminatum; Liver Failure; Primary Sclerosing Cholangitis

Abbreviations: Cholangiopancreatography; PSC: Primary Sclerosing Cholangitis; XD: Xanthoma Disseminatum; MRCP: Magnetic Resonance

\section{ABSTRACT}

Introduction: Xanthoma disseminatum (XD) is a rare, primarily cutaneous nonLangerhans cell histiocytosis. XD characteristically presents as generalized reddishbrown or yellow-colored papules and plaques on the skin often involving the trunk, face, and flexures. While XD is typically benign and is known to remit spontaneously, a handful of case reports have described systemic involvement, which carries a poor prognosis.

Case Presentation: A 21-year-old male with end-stage renal disease presented to the hospital for an unintentional nine-kilogram weight loss over two months as well as eight months of a pruritic papular rash which appeared on the back and generalized centrifugally. Initial laboratory studies suggested a cholestatic liver process. Magnetic resonance cholangiopancreatography (MRCP) was consistent with primary sclerosing cholangitis, but the initial read of a liver biopsy favored primary biliary cirrhosis. Biopsies of cutaneous and colonic lesions revealed histiocytic infiltration without lipid deposition. Re-examination of the liver biopsy specimen also demonstrated histiocytic infiltration, leading to a diagnosis of xanthoma disseminatum.

Conclusions: Xanthoma disseminatum is an exceedingly rare disease that typically presents as a dermatologic process. Even more rarely, it can disseminate, which is usually a fatal process.

\section{Background}

Xanthoma disseminatum (XD) is a rare, primarily cutaneous non-Langerhans cell histiocytosis. XD characteristically presents as generalized reddish-brown or yellow-colored papules and plaques on the skin often involving the trunk, face, and flexures [1]. While XD is typically benign and is known to remit spontaneously, a handful of case reports have described systemic involvement, which carries a poor prognosis [2]. This case is the first to report liver failure in an adult as the result visceral xanthoma disseminatum.

\section{Case Presentation}

A twenty-one-year-old male with history of idiopathic endstage renal disease on hemodialysis presented to the hospital for an unintentional nine-kilogram weight loss over two months. Over the previous eight months, he had also noticed an eruption of purple, itchy bumps starting on the back and generalizing to his entire body, including his face. Review of systems was otherwise positive for decreased appetite and early satiety. The patient's vital signs were normal. His examination was notable for diffuse abdominal tenderness, jaundice, and innumerable generalized purple skin papules Figure 1, some excoriated. A smooth yellow right supraorbital plaque was also observed. Admission laboratory studies were significant for an alkaline phosphatase of $969 \mathrm{IU} / \mathrm{L}$, total bilirubin of $15.2 \mathrm{mg} / \mathrm{dl}$ (12.6 mg/dl direct), AST/ALT of 20/47 $\mathrm{U} / \mathrm{L}$, and an INR of 1.8 (MELD score was 37). Total cholesterol was $448 \mathrm{mg} / \mathrm{dl}$ (HDL <10 mg/dl), and serum triglycerides were $346 \mathrm{mg} / \mathrm{dl}$. His hemoglobin was $11.0 \mathrm{~g} / \mathrm{dl}$, and complete blood count was otherwise unremarkable. Ferritin was $1539 \mathrm{ng} / \mathrm{ml}$, with a total iron binding capacity of 174 g/d $\mathrm{gl}$ and C-reactive protein was $3.3 \mathrm{mg} / \mathrm{L}$. Given the labs were consistent with a significant intrahepatic biliary ductal process, additional workup was undertaken. Anti-mitochondrial, anti-myeloperoxidase, antiproteinase 3 , and anti-smooth muscle antibodies were found to 
be negative. Immunoglobin levels were also within normal limits. Following an unrevealing laboratory evaluation, a magnetic resonance cholangiopancreatography (MRCP) revealed multifocal areas of narrowing in the common bile duct and intrahepatic ducts with segmental areas of mild intrahepatic biliary ductal dilatation, consistent with primary sclerosing cholangitis (PSC) Figure 2.

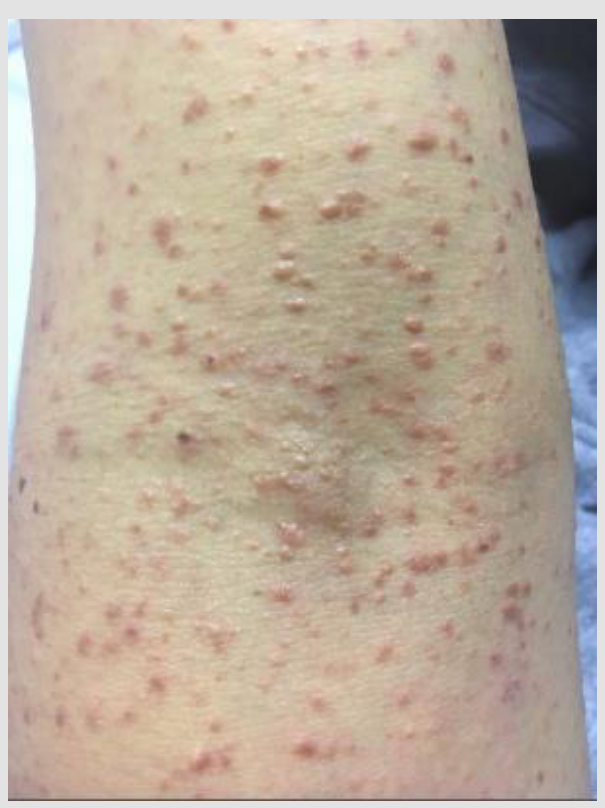

Figure 1: Innumerable red to purple macules and papulonodules on the right antecubital fossa of a twentyone-year-old male.

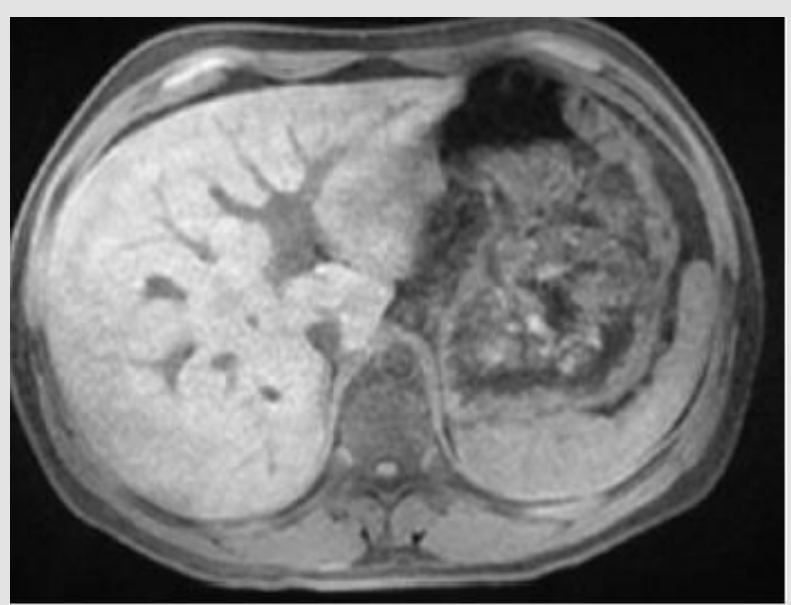

Figure 2: MRCP demonstrating intraheptic biliary dilatation and distortion of the biliary tree.

Liver biopsy was inconclusive, demonstrating cholestasis with mild bile ductular reaction and subtle bile duct injury, with mild portal infiltration of lymphocytes, eosinophils, and neutrophils. There was mild lobular inflammation with significant fibrosis, and poorly formed, non-necrotizing granulomata in the portal triad and lobules. The histopathological differential diagnosis included infection, drug-induced liver injury, or primary biliary cholangitis, less likely PSC. A colonoscopy performed to evaluate for PSCassociated ulcerative colitis disclosed plaques which appeared to be lipid-laden, supporting the diagnosis of PSC. Upon biopsy, colonic and skin lesions revealed no lipid deposition though, casting doubt on the diagnosis of PSC. Instead, both evidenced collections of histiocytes which were CD68(+), CD1a (-), and S100(-) Figure 3. Overall, the disease presentation was felt clinicopathologically consistent with xanthoma disseminatum (XD). Re-examination of the liver biopsy found the same histiocytic infiltration, affirming the diagnosis of disseminated XD.

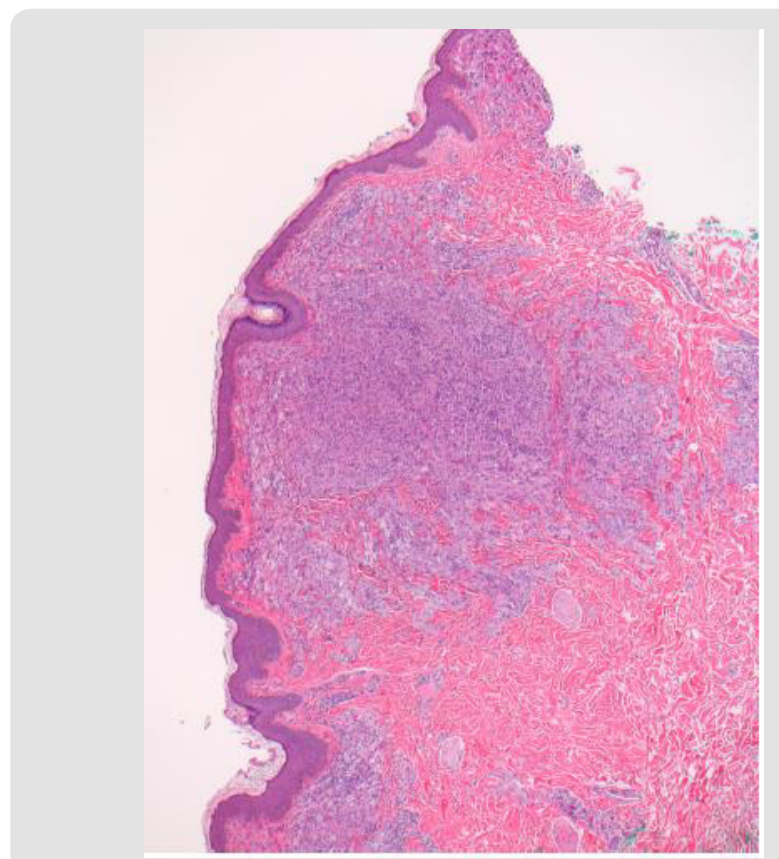

Figure 3: Cutaneous biopsy sample stained with hematoxylin and eosin evidencing a histiocytic infiltrate involving the papillary and reticular dermis.

After consultation with the hematology service, the patient was started on intravenous methylprednisone $50 \mathrm{mg}$ twice a day, while awaiting further molecular and genetic testing. The patient demonstrated mild improvements in hyperbilirubinemia (from $26.0 \mathrm{mg} / \mathrm{dl}$ to $16.0 \mathrm{mg} / \mathrm{dl}$ ) and symptomatology. He was transitioned to oral dexamethasone $10 \mathrm{mg}$ BID, remained hemodynamically stable, and was discharged with outpatient follow-up and plans for eventual chemotherapy. Unfortunately, before he could receive any further treatments, the patient was readmitted to the hospital with worsening exercise tolerance and tachycardia. A diagnosis of sepsis was suspected and paracentesis of a complex loculation of ascites confirmed the diagnosis of spontaneous bacterial peritonitis. $\mathrm{He}$ also received treatment for CMV colitis given radiographic findings, later confirmed on colonoscopy. The patient suffered multiple episodes of melena with hemoglobin levels falling to a nadir of $3.9 \mathrm{mg} / \mathrm{dl}$. During the course of evaluation for a source, the patient became tachypneic with new, bilateral crackles on inspiration. He received bedside dialysis, but symptoms worsened, 
and he became hypotensive and hypoxic. He was intubated and placed on vasopressors for support. Blood cultures grew Serratia species, respiratory cultures grew Pseudomonas species, and broncheoalveolar lavage was positive for Pneumocystis. With worsening hypotension, the patient was no longer able to tolerate any form of dialysis and his acidosis worsened. After a discussion with the patient's family regarding his goals of care, they believed he would not want to be kept alive in this condition without hope of recovery. The patient was made DNR/DNI and was palliatively extubated, soon passing away.

\section{Discussion}

Xanthoma disseminatum is a rare, predominantly cutaneous non-Langerhans cell histiocytosis. Xanthoma disseminatum is so named for its characteristic skin lesions. However, this is a misnomer, as it is a disorder of histiocytes rather than lipid metabolism as in true xanthomata. Since its first description in 1938 , only $\sim 100$ case reports have been published. XD occurs most frequently in men during the third decade of life [3]. It is characterized by reddish-brown to yellow papules and plaques on the skin often involving the trunk, face, and flexural surfaces. One prior case series categorized XD into three distinct clinical patterns [4]. The most common presentation is persistence of a purely dermatologic disease in patients, although the lesions can be cosmetically and functionally inhibiting [5]. Rarely, patients with a primary dermatologic process can have spontaneous regression. And most rarely, XD can disseminate and cause multiorgan dysfunction, which carries a poor prognosis. Multiple patients were found to have central diabetes insipidus as a result of CNS infiltration [6]. One patient was diagnosed at twenty years old with a purely dermatologic presentation, subsequently developed diabetes insipidus at age forty-eight, and was continuing to do well at sixty years old [7]. Individual reports were found on dissemination to multiple other organs. A 37-year-old male required tracheotomy secondary to obstruction from mucous membrane lesions, although he was otherwise well afterwards [8]. Individual cases were also found, typically with young men, involving the kidney, pancreas, eye, and bones [6]. One 72-yearold female was found to have involvement of the skeletal and heart muscle and passed away from ulcerated skin lesions that became infected [9]. One case did describe hepatobiliary involvement, although that was incidentally found and not the primary presentation [6]. Clinicopathological correlation is necessary for distinguishing XD from other histiocytoses. Immunohistochemical staining will evidence CD1a and S100 negativity as well as CD68 positivity [3]. Treatment for systemic XD is derived from prior case reports, and may include immunomodulatory medications e.g., thalidomide, immunosuppressive medications e.g., azathioprine or corticosteroids, and/or chemotherapeutic agents such as clofarabine, cladribine, or cyclophosphamide [10-13].
Based on an extensive literature search, this is the first case of XD presenting primarily with liver failure in an adult, with one similar presentation in a 13-year-old boy [14]. Although hepatic imaging was suggestive of primary sclerosing cholangitis, in our search for further evidence, our initial diagnosis was ill supported by results of cutaneous and colonic biopsies, which unexpectedly revealed histiocytic infiltrates. These findings necessitated re-examination of the liver biopsy, as there was no mention of histiocytes in the initial report. Given the variability of liver biopsy results $[15,16]$, the pathologist needs to understand the clinical scenario in order to determine whether additional stains or techniques or required, as was necessary to confirm the diagnosis of xanthoma disseminatum. Unfortunately, systemic XD is often progressive and fatal, as in this case. Given the paucity of known cases, no trials or guidelines exist to suggest an optimal treatment regimen. Based on previous reportage and our own experience in this case, high-dose systemic corticosteroids likely provide some initial symptomatic relief and can function as a temporizing measure before a steroid sparing regimen is instantiated. One report showed significant regression in skin lesions using thalidomide, with sustained improvement after 3 months [12]. In another report, azathioprine did not result in improvement in cutaneous lesions [3]. Previous cases have suggested the benefit of clofarabine, cladribine, and cyclophosphamide, with some patients showing significant improvement, but only on individual bases [11]. As such, for patients with systemic xanthoma disseminatum, we suggest a trial of highdose steroids, with subsequent chemotherapy based on patient characteristics, side effect profiles, and potential genetic profiling going forward. Of note, this patient had end-stage renal disease of unknown origin for 2 years. Given his young age, and significant systemic involvement of XD, we postulate that his renal disease was possibly secondary to XD. Earlier recognition would have enabled earlier intervention and potentially altered his clinical course.

\section{Conclusions}

Xanthoma disseminatum is an exceedingly rare disease that typically presents as a dermatologic process. Early recognition and referral to a center with experience in treating histiocytoses provides for an optimal outcome. Rarely, it can disseminate to involve visceral organs, as in this case, which is typically fatal.

\section{References}

1. Gong HZ, Zheng HY, Li J (2018) Xanthoma disseminatum. Lancet 391(10117): 251.

2. Pinto ME, Escalaya GR, Escalaya ME, Pinto JL, Chian CA, et al. (2010) Xanthoma disseminatum: case report and literature review. Endocr Pract. 16(6): 1003-1006.

3. Attia AM, Bakry OA, Mohamed EE (2014) Xanthoma disseminatum: A progressive case with multisystem involvement. JPGM 60(1): 69-71.

4. Caputo R, Veraldi S, Grimalt R, R Gianotti, A Tosti, et al. (1995) The various clinical patterns of xanthoma disseminatum. Dermatology 190(1): 19-24. 
5. Varotti C, Bettoli V, Berti E, S Cavicchini, R Caputo et al. (1991) Xanthoma disseminatum: A case with extensive mucous membrane involvement. J Am Acad Dermatol 25(2 pt2): 433-436.

6. Woollons A, Darley CR (1998) Xanthoma disseminatum: a case with hepatic involvement, diabetes insipidus and type IIb hyperlipidemia. Clinical and Experimental Dermatology 23(6): 277-280.

7. Kalz F, Hoffman MM, Lafrance A (1970) Xanthoma disseminatum. Dermatologica 140: 129-141.

8. Seaton ED. Pillai GJ, Chu AC (2004) Treatment of xanthoma disseminatum with cyclophosphamide. BJD 150(2): 346-349.

9. Frank SB, Weidman AI (1952) Xanthoma disseminatum: An Unusual Form with Extension of Xanthomatous Changes into Muscle. AMA Arch Derm and Syph 65(1): 88-94.

10. Yusuf SM, Mijinyawa MS, Musa BM, Mohammed AZ (2008) Xanthoma disseminatum in a black African woman. Int J Dermatol 47(11): 11451147.

11. Picarsic JL, Chikwava K (2019) Disorders of Histiocytes in Hematopathology (Third Edition). 2019 Elsevier.

ISSN: 2574-1241

DOI: 10.26717/BJSTR.2021.34.005539

Maxwell Bressman. Biomed J Sci \& Tech Res

(C) This work is licensed under Creative

Submission Link: https://biomedres.us/submit-manuscript.php
12. Zinoin M, Hali F, Marnissi F, Lazaar S, Benchikhi H, et al. (2015) Xanthoma disseminatum with asymptomatic multisystem involvement. Ann Dermatol Venereol 142(4): 276-280.

13. Buyukavci M, Ayse Selimoglu, Umran Yildirim, Vildan Ertekin, Mustafa Atasoy, et al. (2005) Xanthoma Disseminatum with Hepatic Involvement in a Child. Pediatric Dermatology 22(6): 550-553.

14. Olsson R, I Hägerstrand, U Broomé, A Danielsson, G Järnerot, L Lööf, et al. (1995) Sampling variability of percutaneous liver biopsy in primary sclerosing cholangitis. Journal of Clinical Pathology 48(10): 933-935.

15. Pournik O, Seyed Moayed Alavian, Leila Ghalichi, Bahram Seifizarei, Leila Mehrnoush, et al. (2014) Inter-observer and Intra-observer Agreement in Pathological Evaluation of Non-alcoholic Fatty Liver Disease Suspected Liver Biopsies. Hepat Mon 14(1): e15167.

16. Horvath B, Daniela Allende, Hao Xie, John Guirguis, Jennifer Jeung, et al. (2017) Interobserver Variability in Scoring Liver Biopsies with a Diagnosis of Alcoholic Hepatitis. Alcohol Clin Exp. Res 41(9): 1568-1573.

BIOMEDICAL
RESEARCHES $\quad \begin{aligned} & \text { Assets of Publishing with us } \\ & \text { - Global archiving of articles }\end{aligned}$

